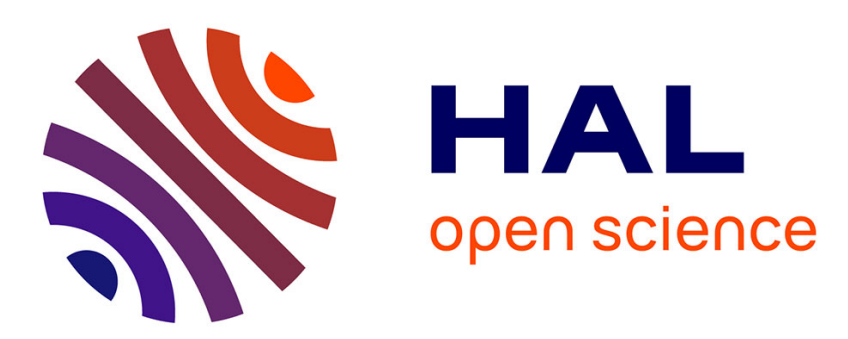

\title{
Brouillards dans les mélanges vapeur gaz incondensable
} Thierry Poinsot, J. Huetz

\section{To cite this version:}

Thierry Poinsot, J. Huetz. Brouillards dans les mélanges vapeur gaz incondensable. Revue de Physique Appliquée, 1985, 20 (3), pp.163-172. 10.1051/rphysap:01985002003016300 . jpa-00245319

\section{HAL Id: jpa-00245319 https://hal.science/jpa-00245319}

Submitted on 1 Jan 1985

HAL is a multi-disciplinary open access archive for the deposit and dissemination of scientific research documents, whether they are published or not. The documents may come from teaching and research institutions in France or abroad, or from public or private research centers.
L'archive ouverte pluridisciplinaire HAL, est destinée au dépôt et à la diffusion de documents scientifiques de niveau recherche, publiés ou non, émanant des établissements d'enseignement et de recherche français ou étrangers, des laboratoires publics ou privés. 


\title{
Brouillards dans les mélanges vapeur gaz incondensable
}

\author{
T. Poinsot et J. Huetz \\ C.N.R.S. G.R. 14, Ecole Centrale de Paris, 92290 Châtenay-Malabry, France
}

(Reçu le 26 juin 1984, révisé le 9 novembre, accepté le 16 novembre 1984)

Résumé. - Cette étude met en évidence les domaines d'apparition de brouillards lors de la condensation d'un mélange vapeur d'eau gaz incondensable sur une paroi froide. Les résultats expérimentaux sont comparés avec ceux d'un modèle théorique qui permet le calcul local des sursaturations au sein du mélange vapeur gaz pour un cas simple monodimensionnel. L'influence des incondensables apparaît comme déterminante, non seulement sur le flux thermique à la paroi (effet classique de blocage de la condensation par l'accumulation des incondensables sur la paroi froide) mais aussi sur l'état thermodynamique de la vapeur au sein du mélange, c'est-à-dire sur l'apparition de gouttelettes dans le mélange. Un critère de formation de ces brouillards est proposé pour le cas étudié.

\footnotetext{
Abstract. - Condensation of a vapour gas mixture on a cold surface is studied experimentally. Results indicate that a non-condensable gas influences condensation in two ways :

- it diminishes the wall heat flux by reducing diffusion rates;

- it may trigger homogeneous nucleation of vapour in the mixture.

A numerical study of the thermodynamic state of water vapour in a mixture of vapour and non-condensable gas shows that parameters governing the appearance of fogs are the mean mass fraction of non-condensable gas and the vapour temperature. The effects of these parameters are investigated and a criterion of fog appearance is proposed.
}

\section{Nomenclature.}

$a \quad$ Constante $=M /(\rho . D)$ (Eq. (17))

$D \quad$ Coefficient de diffusion binaire vapeur gaz (ou gaz vapeur)

$g \quad$ Accélération de la pesanteur

I Taux de formation des noyaux liquides au cours d'une nucléation homogène

$k \quad$ Constante de Boltzmann

$K \quad$ Constante fonction des fréquences de collision des molécules de vapeur

$M_{1} \quad$ Masse atomique de la vapeur

$M_{2}$ Masse atomique du gaz incondensable

$M \quad$ Débit masse de vapeur par unité d'aire (Eq. (6))

$\mathfrak{L} \quad$ Chaleur de vaporisation de l'eau

$l \quad$ Distance entre parois

$P_{1} \quad$ Pression partielle locale de vapeur

$P_{S}(\theta)$ Pression locale de saturation à la température $\theta$

$P \quad$ Pression totale

$s \quad$ Rapport de sursaturation locale (Eq. (21))

$S_{\mathrm{c}} \quad$ Rapport de sursaturation critique (Eq. (22))

$V_{1}, V_{2}$ Vitesses macroscopiques de la vapeur, du gaz incondensable
$V \quad$ Vitesse barycentrique moyenne (Eq. (5))

$W \quad$ Fraction massique de gaz incondensable (Eq. (2))

$W_{\mathrm{m}} \quad$ Valeur moyenne de $W$ dans la cavité (Eq. (1)).

Symboles grecs

$\alpha \quad$ Diffusivité thermique du mélange vapeur gaz

$\lambda \quad$ Conductivité du mélange vapeur gaz

$\rho \quad$ Masse volumique totale (Eq. (3))

$\rho_{1} \quad$ Masse volumique de la vapeur

$\rho_{2} \quad$ Masse volumique du gaz incondensable

$\theta(0)$ Température de la paroi poreuse $(x=0)$

$\theta(l) \quad$ Température de la paroi froide $(x=l)$

$\theta(x) \quad$ Température locale

$\theta_{\mathrm{s}} \quad$ Température de saturation correspondant à la pression partielle moyenne de vapeur dans la cavité

$\Delta \theta \quad$ Surchauffe de la vapeur en $x=0$ (Eq. (15))

$\Phi \quad$ Flux thermique à la paroi $x=l$ (Eq. (13))

$\Phi_{\mathrm{c}} \quad$ Flux conductif à la paroi $x=l$ (Eq. (14)).

Indice :

$\dot{\mathrm{m}}=$ valeurs moyennes dans la cavité. 


\section{Introduction.}

Les processus de condensation en présence de gaz incondensables ont été largement abordés au cours des dernières années [1 à 4]. La réduction du flux thermique entraînée par l'accumulation de gaz incondensables au voisinage des parois froides a été étudiée en convection forcée de manière théorique [5] et expérimentale [6].

Les problèmes de convection naturelle le long d'une plaque verticale ont été résolus numériquement [7-9] et approximativement (méthode des profils) [10] pour des condensations en film et des mouvements dus aux différences de fractions massiques en gaz incondensable dans le mélange vapeur gaz. Cette étude a pour but de faire apparaître une complexité supplémentaire, le plus souvent négligée par les modèles de condensation avec incondensables, surtout.lorsque ceux-ci sont en proportion importante : la condensation de la vapeur peut se produire sur la paroi froide (modes classiques de condensation en film ou en gouttes) mais aussi en même temps au sein du mélange. Le choix du type de condensation - à la paroi ou en volume - n'est pas évident et résulte des conditions thermiques imposées au mélange surchauffe, fraction moyenne de gaz incondensable, etc.

1.1. - Nous présenterons des résultats expérimentaux obtenus au cours d'une étude plus générale des transferts thermiques dans une enceinte cylindrique à axe horizontal contenant de l'azote dans laquelle on injecte latéralement de la vapeur pure(Fig. 1). Cette expérience a permis de constater l'existence de brouillards au sein du mélange et de déterminer quel ensemble de facteurs en provoque l'apparition. De plus, elle a fourni pour la géométrie envisagée les paramètres caractéristiques de formation et de disparition des brouillards ainsi que la description de leur couplage avec un autre processus déterminant lors de l'étude des mélanges vapeur gaz : la déviation de l'écoulement par les forces d'Archimède. Ce couplage peut introduire de fortes oscillations de la pression de l'enceinte

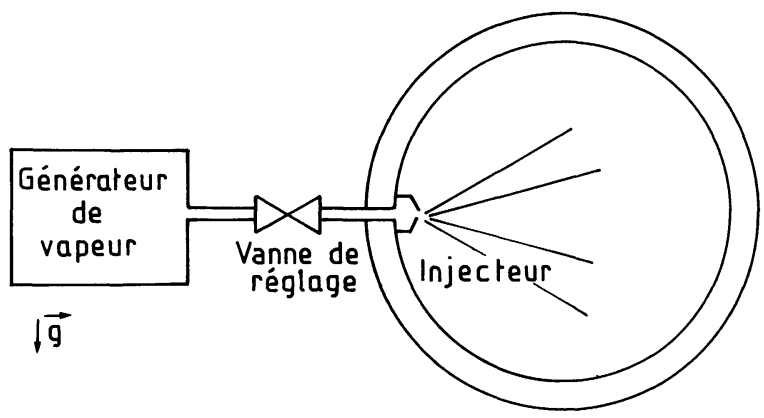

Fig. 1. - Schéma expérimental. Le cylindre à axe horizontal contient une quantité imposée d'azote. Au temps $t=0$, la vanne est ouverte et met en communication le générateur de vapeur et le cylindre.

[Experimental apparatus.] et de la vitesse de la vapeur injectée (en module et en direction). Ce type de phénomène intervient par exemple dans les problèmes de sûreté des centrales nucléaires lors de la rupture du circuit primaire dans l'enceinte de confinement, qui contient initialement de l'air.

1.2. - Les expériences indiquent que l'alternative condensation à la paroi ou condensation en brouillards est déterminée par la surchauffe de la vapeur et par la quantité d'incondensables présents dans l'enceinte et le flux thermique que la paroi peut évacuer. Nous établirons une théorie simplifiée, limitée au cas unidimensionnel en régime permanent et montrerons comment ce type de condensation peut être prévu par un calcul de sursaturation en tout point de l'écoulement, ce qui nécessite un calcul local des fractions massiques et des températures.

\section{Mise en évidence expérimentale et évolution des brouillards.}

L'étude des condensations avec brouillards n'était pas le premier objectif de l'expérimentation présentée. Celle-ci a tout d'abord servi à visualiser le mélange et la trajectoire d'un jet de vapeur d'eau, ainsi qu'à établir les échanges thermiques de celui-ci avec les parois, dans une enceinte cylindrique contenant de l'azote (Fig. 2). Cette configuration expérimentale est plus complexe que celle des chambres de diffusion habituellement employées pour l'étude de l'apparition de brouillards dans une vapeur pure et la détermination des taux de sursaturation critique [13-15]. En contrepartie, elle présente les avantages suivants :

- elle se rapproche de nombreux cas pratiques [11];

- elle fait apparaître le processus complet de formation et de disparition des brouillards;

- elle met en évidence la possibilité d'un couplage entre l'injection de vapeur et la formation des brouillards.

2.1 DesCription. - L'injection de vapeur se fait par une fente située le long d'une génératrice du cylindre et munie d'un système de tranquillisation afin d'assurer un caractère bidimensionnel du jet dans la zone d'observation optique (Fig. 3). La visualisation est réalisée par strioscopie. Des thermocouples sont placés dans un manchon fluxmètre qui tapisse l'intérieur du cylindre. Celui-ci est initialement rempli d'azote (pression $P_{0}$ ) et plongé dans de l'air à température ambiante. A partir du temps $t=0$, la vanne est ouverte et met en communication le générateur de vapeur (pression $\boldsymbol{P}_{\mathbf{g}}$ ) et l'enceinte. Après une phase de remplissage, où le jet est turbulent et où la pression dans l'enceinte passe d'une valeur $P_{0}$ à une valeur proche de $P_{g}$, la vitesse d'injection diminue et le jet devient laminaire. En régime stationnaire, le débit de vapeur injectée compense le débit condensé sur les parois. La déviation du jet de vapeur due aux différences entre la masse volumique de la vapeur injectée 


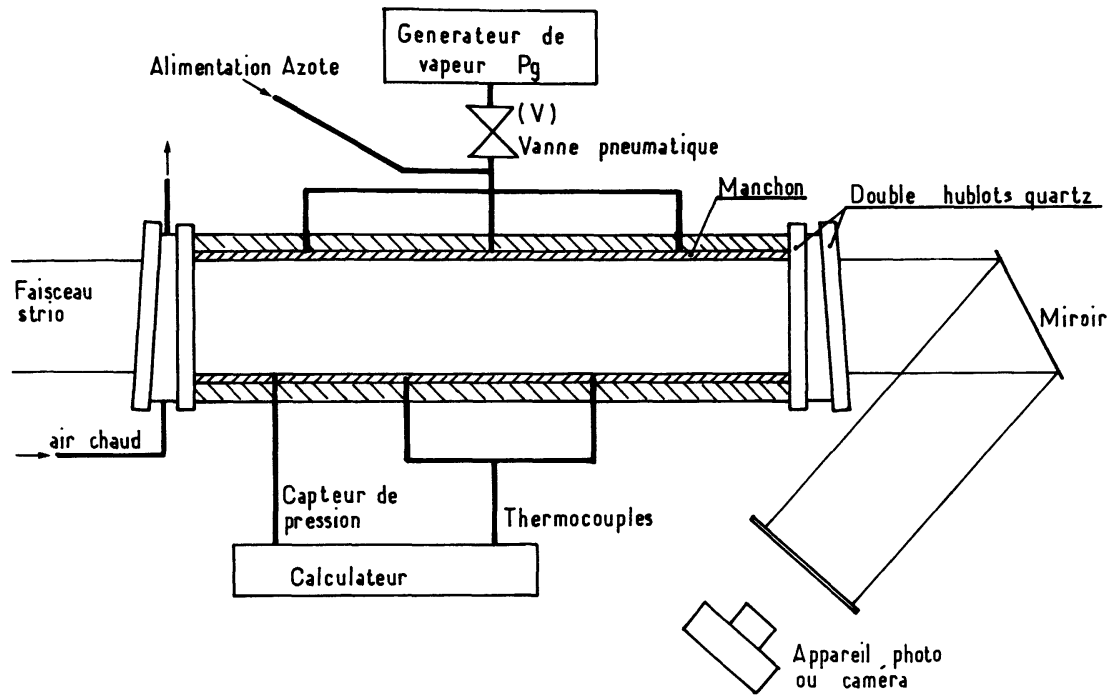

Fig. 2. - Schéma expérimental. Acquisition des données.

[Data acquisition.]
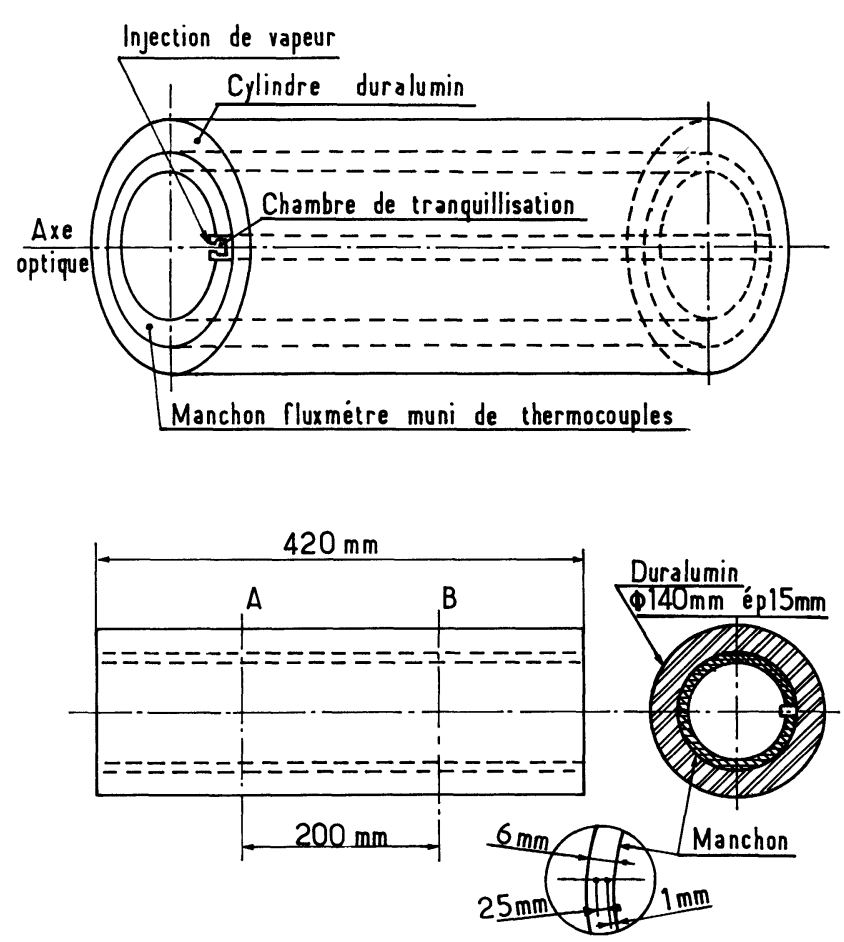

Fig. 3. - Cylindre d'expérimentation avec son dispositif d'injection. Positionnement des thermocouples dans le manchon.

[Experimental cell and vapour injector.]

et celle du mélange vapeur gaz devient d'autant plus forte que la vitesse d'injection générée par une pression amont constante est faible. Finalement, l'écoulement de la vapeur devient quasiment vertical depuis l'injecteur jusqu'à la partie supérieure du cylindre - ceci n'est vérifié que pour des jets confinés dans une enceinte dont les parois sont faiblement conductrices de la chaleur et ne créent donc, en régime permanent, qu'un faible appel de vapeur. (La résistance thermique du manchon utilisé est $\left.4 \times 10^{-2} \mathrm{Km}^{2} / \mathrm{W}\right)$. La strioscopie montre que le mélange occupant la partie basse du cylindre est alors presque immobile. Les thermocouples disposés dans le manchon et dans le mélange vapeur gaz indiquent une différence de température haut/bas comprise entre 20 et $50^{\circ} \mathrm{C}$. Bien que ni la température du fond de l'enceinte, ni celle du sommet ne soient rigoureusement constantes, l'écart moyen de température haut bas reste sensiblement constant pour une expérience, un taux d'incondensables et une surchauffe de la vapeur donnés. L'évolution du système à partir de l'état décrit ci-dessus se fait de deux façons selon les conditions thermiques imposées :

- Pour des surchauffes élevées de la vapeur injectée ou de faibles valeurs de la fraction massique moyenne d'azote $W_{\mathrm{m}}$ dans l'enceinte, la différence de température entre le haut et le bas du cylindre est faible (environ $20^{\circ} \mathrm{C}$ ). Aucune condensation n'apparaît dans le mélange, la déviation du jet reste constante et le système est stable (Fig. 4).

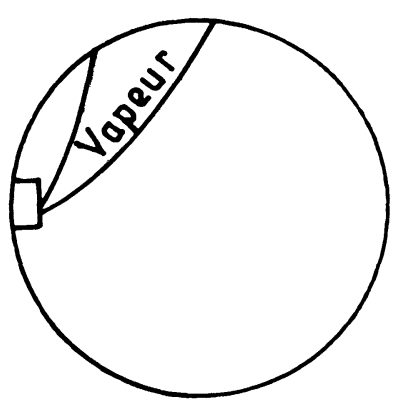

Fig. 4. - Ecoulement dévié stable. Le mélange est sec. [Stable flow without fog.] 
- Pour des surchauffes faibles ou des valeurs élevées de $W_{\mathrm{m}}$, la différence de température entre haut et bas est importante (environ $50^{\circ} \mathrm{C}$ ). Le mélange présent au fond de l'enceinte devient sursaturé au-delà du seuil critique et la nucléation homogène se déclenche en créant un brouillard (Fig. 5). La chute de pression corrélative entraîne un appel de vapeur donc une accélération du jet qui, de ce fait, dévie moins et repousse le brouillard (Fig. 6). La pression remonte, la vitesse du jet diminue à nouveau et sa déviation augmente (Fig. 7). Après quelques secondes, l'écoulement redevient semblable à celui de la figure 4 : le système génère des oscillations. Leur période est d'environ dix secondes dans les conditions expérimentales utilisées.

Cette période est la somme des temps caractéristiques suivants :

- temps de déviation du jet à partir d'un état non dévié : ce calcul est effectué dans [11] et donne des temps de l'ordre d'une à deux secondes pour des fractions moyennes $W_{\mathrm{m}}$ de 0,5 ;

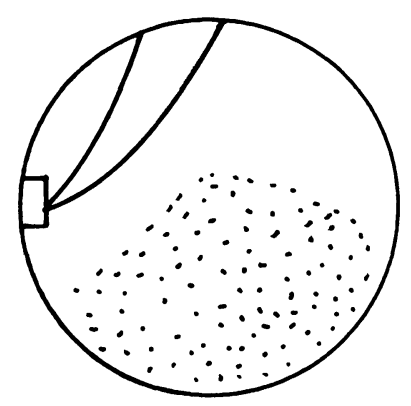

Fig. 5. - Au fond du cylindre, le mélange vapeur gaz devient lumineux. La vapeur sursaturée s'est condensée et les gouttes diffusent le faisceau de strioscopie.

[Supersaturation in the lower part of the cell triggers condensation in the mixture.]

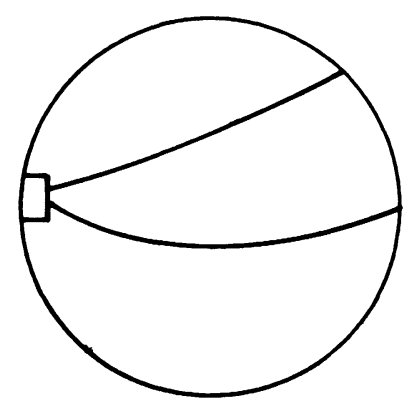

Fig. 6. - La chute de pression causée par la condensation en brouillard provoque une accélération du jet de vapeur qui est moins dévié et repousse le brouillard sur les parois du cylindre.

[The vapour jet is accelerated by the pressure drop in the cell.]



Fig. 7. - Après disparition du brouillard la pression augmente, la vitesse du jet diminue et sa déviation redevient forte.

[The fog disappears, the pressure increases and the jet deviates again.]

- temps d'établissement des profils de température et de fractions massiques dans la zone basse du cylindre : à partir de la déviation totale du jet, la sursaturation locale augmente dans les zones qui ne reçoivent plus de vapeur. Lorsque cette sursaturation atteint la valeur critique nécessaire au déclenchement de la nucléation homogène [13], le brouillard apparaît ;

- temps de réponse des tuyauteries de vapeur (en général négligeable).

Les figures 8 et 9 représentent les variations de la pression totale et de la température dans le mélange vapeur gaz au fond de l'enceinte. On remarque que la

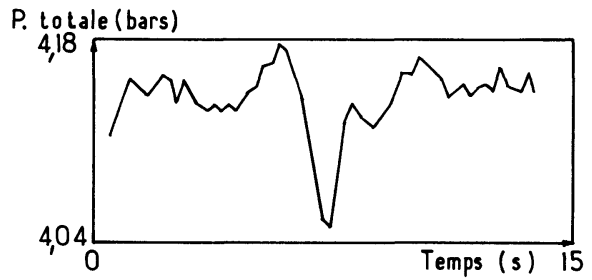

Fig. 8. - Variation de la pression totale au cours d'une condensation en brouillard.

[Total pressure variation during for apparition.]

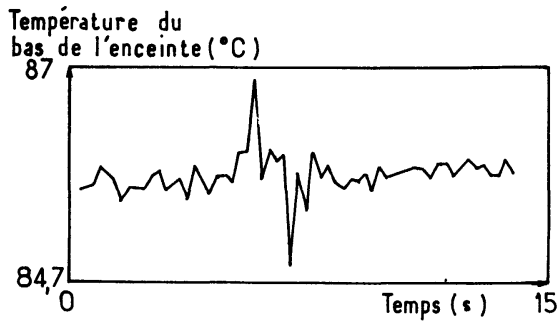

Fig. 9. - Variation de la température d'un thermocouple situé dans le bas de l'enceinte au cours d'une condensation en brouillard.

[Temperature variation of a thermocouple located in the lower part of the cell during fog apparition.] 
température indiquée par un thermocouple prolongé dans une vapeur sursaturée est certainement plus proche de la température de saturation de cette vapeur que de la température réelle. Cette mesure conduit, en fait, à une estimation de la pression partielle locale de vapeur d'eau. Ce résultat est confirmé par des mesures simultanées de la pression totale et de la température du thermocouple situé dans le bas du cylindre : au cours d'une condensation en brouillard, la pression totale et la température du thermocouple diminuent : cette dernière varie donc comme la pression partielle de vapeur qui chute pendant la condensation et non comme la température vraie, qui est constante ou augmente (Fig. 10). En effet, au cours d'une condensation en brouillard, la vapeur passe d'un état sursaturé $\mathrm{A}$ à un état $\mathrm{B}$ situé sur la courbe de saturation. La position exacte de $B$ dépend des conditions thermiques au voisinage de la zone de condensation. Cependant, $B$ est situé au-delà de $B_{0}$ sur la courbe de saturation. ( $B_{0}$ correspond à un état saturé à une température égale à celle du point $\mathrm{A}$ ).

\section{Critère d'apparition des brouillards dans un cas simplifié.}

Bien qu'il ne soit pas envisageable de quantifier l'ensemble des phénomènes intervenant dans l'expérimentation présentée au 2., il est possible de trouver un schéma simple représentant de façon approchée les domaines d'apparition d'une condensation en brouillard et correspondant avec les résultats expérimentaux. En effet, dans la zone basse du cylindre, le mélange vapeur gaz est presque immobile (Fig. 4) et les transferts qui s'y produisent essentiellement diffusifs. Cette phase de l'écoulement peut être étudiée par un modèle schématisé sur la figure 11. Dans l'espace compris entre deux plans infinis $x=0$ et $x=l$, la paroi supérieure est supposée poreuse et laisse entrer de la vapeur pure de façon à maintenir la pression totale constante dans la cavité. Cette paroi est assimilée à la frontière inférieure du jet de vapeur. La condensation a lieu sur la paroi en $x=l$ qui correspond au fond du cylindre. Le principe du calcul est que les profils de sursaturation réels dans le cylindre entre deux points comme $P_{0}$ et $P_{1}$ sont semblables à ceux calculés entre $P_{p_{0}}$ et $P_{p_{1}}$ (Fig. 11). Pour cela, les températures $\theta(0)$ et $\theta(l)$ sont imposées et choisies égales à celles mesurées au cours des expériences. De plus, on impose la fraction massique moyenne de gaz incondensable $W_{\mathrm{m}}$ dans la cavité définie par :

$$
W_{\mathrm{m}}=1 / l \int_{0}^{l} W(x) \mathrm{d} x
$$

où $W$ est la fraction massique de gaz incondensable :

$$
W=\rho_{2} / \rho
$$

et $\rho_{2}$ et $\rho$ sont les masses volumiques respectives du gaz incondensable et du mélange vapeur gaz.

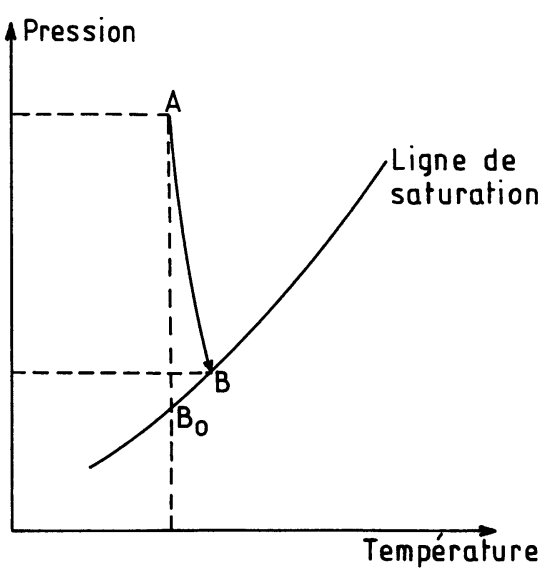

Fig. 10. - Evolution thermodynamique de la vapeur au cours d'une condensation en brouillard (passage d'un état sursaturé $A$ à un état saturé B). La position précise du point $B$ dépend des échanges thermiques de la vapeur avec le milieu qui l'entoure. Dans tous les cas, la température de B est supérieure à celle de $\mathrm{A}$.

[Thermodynamical state of vapour during fog condensation.]

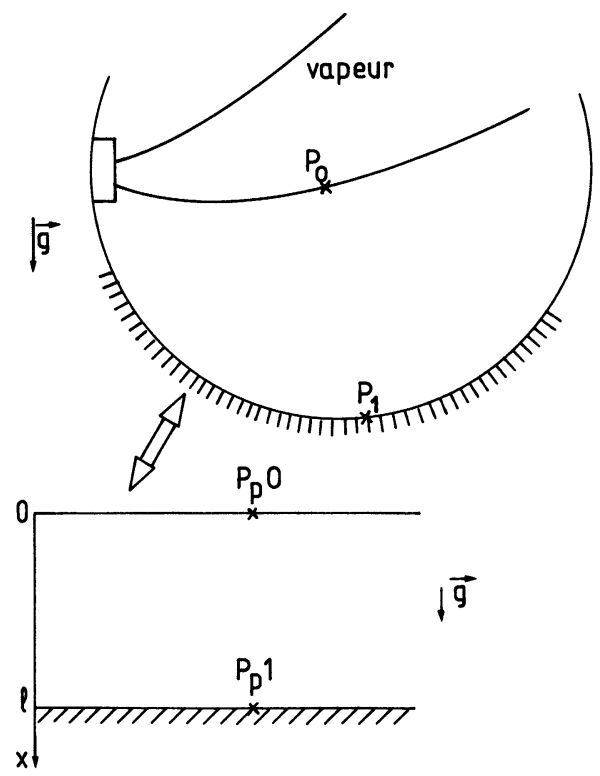

Fig. 11. - Modélisation des transferts thermiques menant à l'apparition d'un brouillard dans la zone basse du cylindre. La paroi en $x=0$ est poreuse et laisse entrer la vapeur d'eau. La condensation a lieu sur la paroi en $x=l$.

[Model for heat and species transfer mechanisms leading to fog condensation.]

$\rho_{1}$ est la masse volumique de la vapeur et on a :

$$
\rho=\rho_{1}+\rho_{2} .
$$

On suppose que la masse atomique $M_{2}$ du gaz incondensable est supérieure à celle $M_{1}$ de la vapeur, afin d'éviter tout mouvement de convection naturelle thermique (Fig. 11). Le problème simplifié est alors 
unidimensionnel et on se limite à l'étude du régime permanent. Le calcul est effectué pour une condensation sans brouillard, donc ayant lieu uniquement à la paroi $x=l$.

Si la sursaturation locale ne dépasse pas la sursaturation critique, le régime sera stable. Dans le cas contraire, des brouillards apparaîtront. Enfin, comme le montrent les résultats, pour des fractions massiques moyennes $W_{\mathrm{m}}$ variant entre 0,1 et 0,8 , les flux thermiques sont faibles et la principale résistance thermique est due à la diffusion de la vapeur à travers les incondensables. On vérifie aisément que la résistance thermique des condensats liquides est petite et que leur influence thermique sur le transfert global est négligeable [11].

Les variables à déterminer sont la température $\theta(x)$ et la fraction massique $W(x)$ locales ainsi que le débit de vapeur. Les équations utilisées sont les équations de conservation de la masse d'incondensables, de la masse totale et de la chaleur :

$$
\frac{\partial}{\partial x}(\rho V W)=\frac{\partial}{\partial x}\left(\rho D \frac{\partial}{\partial x} W\right)
$$

où $W$ est donné par l'équation (2) et $V$ est la vitesse moyenne locale définie par

$$
V=\left(\rho_{1} V_{1}+\rho_{2} V_{2}\right) / \rho
$$

$D$ est le coefficient de diffusion binaire vapeur gaz et $V_{1}$ et $V_{2}$ sont les vitesses respectives de la vapeur et de l'incondensable. Dans le cas du régime permanent $V_{2}=0$ et on a :

$$
M=\rho \cdot V=\rho_{1} V_{1}
$$

où $M$ est le débit masse surfacique de vapeur

$$
V \cdot \frac{\partial \theta}{\partial x}=\frac{1}{\rho C_{\mathrm{p}}} \cdot \frac{\partial}{\partial x}\left(\lambda \frac{\partial \theta}{\partial x}\right)
$$

où $\alpha$ est la diffusivité thermique du mélange

$$
\alpha=\lambda / \rho C_{\mathbf{p}} .
$$

\section{1 CONDITIONS LIMITES ET DONNÉES.}

- Les températures $\theta(0)$ et $\theta(l)$ sont imposées.

- La fraction massique moyenne $W_{\mathrm{m}}$ est imposée (Eq. (1)).

- Sur la paroi $x=l$, la vapeur est saturée.

Donc la pression partielle de vapeur y est égale à la pression de saturation correspondant à la température $\theta(l)$.

$$
P_{1}=P s(\theta(l))
$$

où d'après la loi des gaz, on a :

$$
P_{1}=P \cdot(1-W(l)) /\left(1-\left(1-M_{1} / M_{2}\right) \cdot W(l)\right) .
$$

Le problème est alors entièrement déterminé et le calcul doit fournir le profil de fraction massique
$W(x)$, de température $\theta(x)$, le débit de vapeur $M$ et le flux thermique à la paroi $x=l$ :

$$
\Phi=M \mathcal{L}+\Phi_{\mathrm{c}}
$$

avec

$$
\Phi_{\mathrm{c}}=-\lambda \frac{\partial \theta}{\partial x}(x=l)
$$

Le deuxième terme de l'équation (13), qui représente le flux conductif $\Phi_{\mathrm{c}}$ à la paroi $x=l$ est le plus souvent négligé dans les études de condensation car il est faible devant les flux condensés. Dans le cas présent, cependant, sa contribution peut devenir non négligeable puisqu'il n'existe aucun mouvement parallèle à la paroi, ce qui élimine tout effet convectif et qu'une quantité importante de gaz incondensable limite considérablement la diffusion de la vapeur donc le flux condensé. Afin de faciliter la compréhension des résultats, on introduit la surchauffe de la vapeur en $x=0$ :

$$
\Delta \theta=\theta(0)-\theta_{\mathrm{s}}
$$

où $\theta_{\mathrm{s}}$ est la température de saturation correspondant à la pression partielle moyenne de vapeur d'eau dans la cavité.

3.2 RÉSOlution. - Le système (3), (6) et (7), assorti des conditions (9), (10), (11) admet une solution simple si l'on suppose que la masse volumique du mélange varie peu $\left(\rho=\rho_{\mathrm{m}}\right)$. Dans ce cas, on obtient :

1)

$$
W(x)=a \cdot l W_{\mathrm{m}} \cdot \frac{\exp (a x)}{\exp (a l)-1}
$$

où $a$ est donné par

$$
a=M /\left(D_{\mathrm{m}} \cdot \rho_{\mathrm{m}}\right)
$$

et $D_{\mathrm{m}}$ est la valeur moyenne du coefficient de diffusion. 2)

$$
\begin{aligned}
\theta(x)= & \frac{\theta(l)-\theta(0) \exp \left(a l D_{\mathrm{m}} / \alpha_{\mathrm{m}}\right)}{1-\exp \left(a l D_{\mathrm{m}} / \alpha_{\mathrm{m}}\right)}+ \\
& \quad+\frac{\theta(0)-\theta(l)}{1-\exp \left(a l D_{\mathrm{m}} / \alpha_{\mathrm{m}}\right)} \cdot \exp \left(\frac{a l D_{\mathrm{m}}}{\alpha_{\mathrm{m}}} \cdot \frac{x}{l}\right) .
\end{aligned}
$$

Les valeurs moyennes $\rho_{\mathrm{m}}, D_{\mathrm{m}}, \alpha_{\mathrm{m}}$ de $\rho, D, \alpha$ sont estimées pour une fraction massique égale à $W_{\mathrm{m}}$.

3) Le débit $M$ de vapeur (Eq. (5)) est déterminé par itérations de façon à satisfaire l'équation (11) en utilisant les profils donnés par les équations (16) et (18).

Remarque : On montre que l'hypothèse $\rho=\rho_{\mathrm{m}}$ n'introduit pas d'erreur appréciable sur les résultats : le système (3), (6), (7) peut être résolu numériquement en prenant en compte les variations de la masse volumique avec la température et la fraction massique de gaz incondensable [11]. L'écart entre les deux calculs est inférieur à $2 \%$ sur le flux thermique total $\Phi$ à la paroi $x=l$ (Eq. (13)). 
Le flux total s'écrit alors :

$$
\Phi=M \cdot \mathfrak{L}-\lambda(\theta(0)-\theta(l)) \cdot \frac{a \cdot D_{\mathrm{m}}}{\alpha_{\mathrm{m}}} \cdot \frac{1}{\exp \left(-\frac{a D_{\mathrm{m}} l}{\alpha_{\mathrm{m}}}\right)-1} .
$$

De plus, en chaque point, il est possible d'évaluer le rapport $s$ de sursaturation par :

$$
\begin{aligned}
& s=P_{1}(x) / P(x)=P / P S(\theta(x)) \times \\
& \times(1-W(x)) /\left(1-\left(1-M_{1} / M_{2}\right) . W(x)\right)
\end{aligned}
$$

$s$ est égal à 1 en $x=l$.

\subsection{Résultats.}

- Influence de la conduction.

La figure 12 donne les valeurs du flux total $\Phi$ (Eq. (14)) et du rapport du flux conductif $\Phi_{c}$ (Eq. (13))

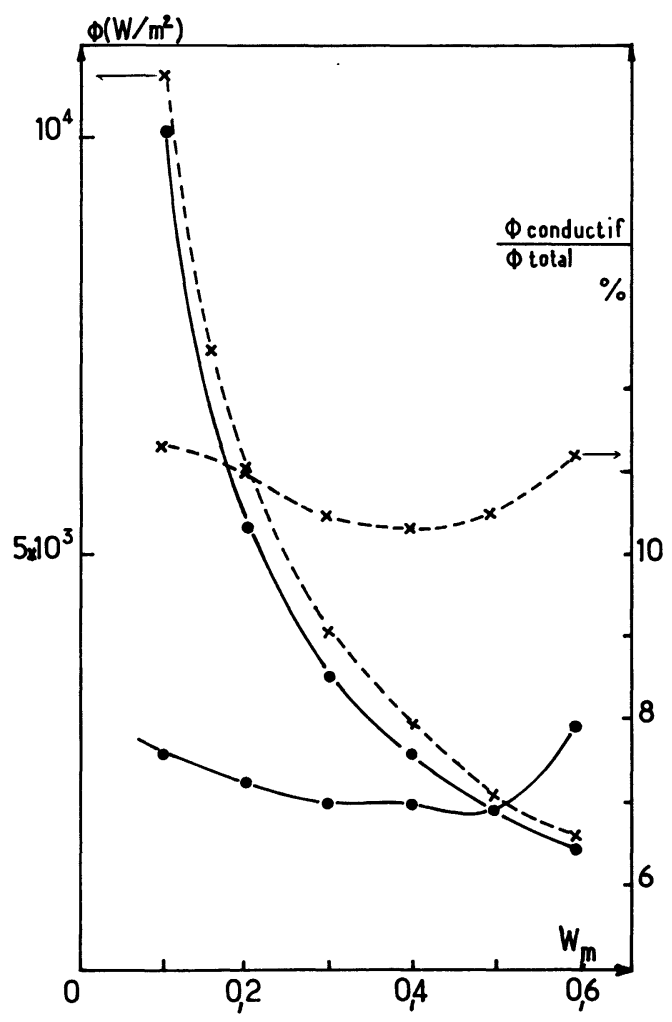

Fig. 12. - Variations du flux total $\Phi$ (Eq. (13)) et du rapport $\Phi_{\mathrm{c}} / \Phi$ (Eq. (14)) en fonction de la fraction massique moyenne $W_{\mathrm{m}}$ d'incondensables (Eq. (1)).

$\theta(0)=100^{\circ} \mathrm{C} \Delta \theta=0{ }^{\circ} \mathrm{C} \theta(l)=50^{\circ} \mathrm{C}$ Courbe en trait plein $\theta(0)=150^{\circ} \mathrm{C} \Delta \theta=50^{\circ} \mathrm{C} \theta(l)=50^{\circ} \mathrm{C}$ Courbe en pointillés.

Dimension de la cavité : $l=0,05 \mathrm{~m}$.

[Variations of heat flux $\Phi$ and ratio $\Phi_{c} / \Phi$ versus mean mass fraction of non-condensable gas.

$\theta(0)=100^{\circ} \mathrm{C} \quad \Delta \theta=0^{\circ} \mathrm{C} \quad \theta(l)=50^{\circ} \mathrm{C}$ Solid line $\theta(0)=150^{\circ} \mathrm{C} \quad \Delta \theta=50^{\circ} \mathrm{C} \quad \theta(l)=50^{\circ} \mathrm{C}$ Dotted line.

Cell dimension $: l=0.05 \mathrm{~m}$.] au flux total pour une surchauffe de 0 ou $50^{\circ} \mathrm{C}$ en fonction de la fraction massique moyenne $W_{\mathrm{m}}$. On retrouve la décroissance rapide du flux total lorsque $W_{\mathrm{m}}$ augmente. Même en diffusion pure et en l'absence de convection, la conduction reste un facteur secondaire devant la condensation à la paroi. Il est clair que, dans le cas de condensation avec brouillard, ce résultat doit être corrigé, car l'énergie dégagée par condensation au sein du mélange vapeur gaz ne peut plus alors migrer vers la paroi que par conduction. La conduction devient un phénomène prépondérant dans le transfert et la modélisation correspondante doit la faire intervenir.

- Influence des dimensions de la cavité.

Les équations (16) et (18) montrent que le groupement $a . l$ ne dépend que des valeurs données à $\theta(0)$, $\theta(l)$ et $W_{\mathrm{m}}$. Dans la mesure où le flux conductif est faible devant le flux de condensation $M$, on peut affirmer que le flux total varie de façon inversement proportionnelle à $l$.

- Influence de $W_{\mathrm{m}}$ (Eq. (1)).

La figure 13 donne les courbes $\Phi=F(\theta(0)-\theta(l))$ pour diverses valeurs de $W_{\mathrm{m}}$ et une surchauffe nulle.

- Existence d'une zone sursaturée.

Une hypothèse importante du modèle est l'absence de condensation ailleurs qu'à la paroi froide $(x=l)$.

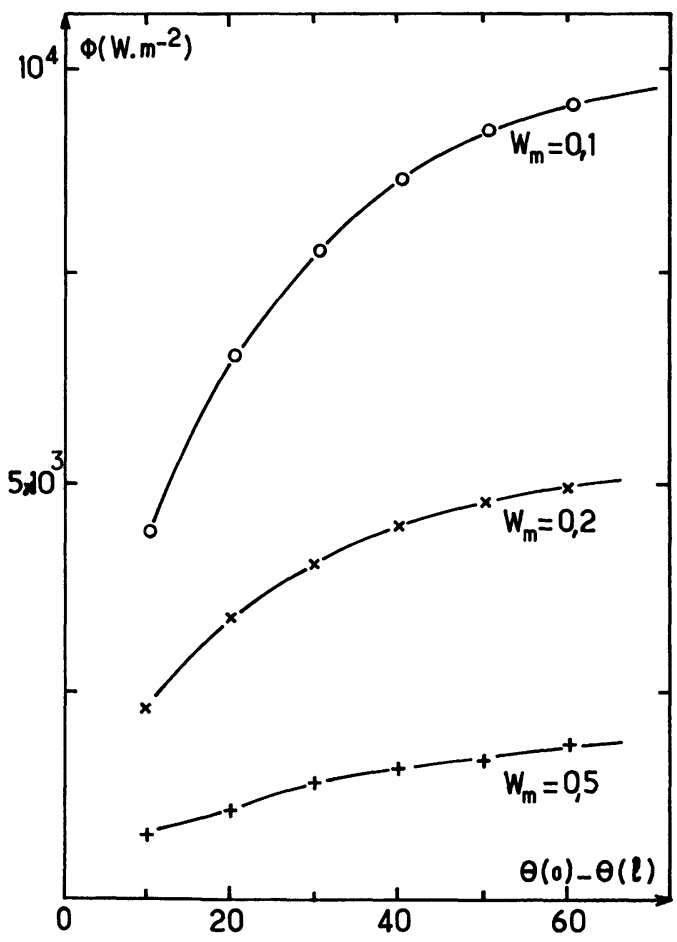

Fig. 13. - Variations du flux total avec l'écart de température entre les parois. La température en $x=0$ est imposée : $\theta(0)=100^{\circ} \mathrm{C}$. Les courbes sont paramétrées en fonction de la fraction massique moyenne $W_{\mathrm{m}}$ (Eq. (1)).

[Heat flux variations versus temperature drop between cell walls. $\theta(0)=100^{\circ} \mathrm{C}$. Parameter : the mean mass fraction of non-condensable gas $W_{\mathrm{m}}$ (Eq. (1)).] 
Cette hypothèse n'est pas toujours vérifiée comme le montre la figure 14. Celle-ci représente pour une valeur de la fraction massique moyenne $W_{\mathrm{m}}=0,5$ (Eq. (1)), les variations selon l'abscisse du taux de sursaturation $s$, rapport de la pression partielle locale de vapeur à la pression locale de saturation (calculée à partir de la température locale) :

$$
s=P_{1}(x) / P s(\theta(x)) .
$$

Pour déterminer si l'état thermodynamique de la vapeur peut ou non mener à une condensation au sein du mélange, il faut comparer $s$ (Eq. (21)) avec $S_{\mathrm{c}}$ taux de sursaturation critique limite du déclenchement de la nucléation homogène. Si $s$ est supérieur à $S_{\mathrm{c}}$, la vapeur sèche sera dans un état instable et un brouillard apparaîtra [12]. En fait, le calcul précis de $S_{\mathrm{c}}$ est rendu difficile car la présence d'incondensables peut entraîner une nucléation hétérogène ainsi que l'ont montré les expériences réalisées dans les chambres de diffusion [13-15]. Il est cependant certain que le taux critique correspondant à une vapeur pure immobile (à une pression égale à la pression partielle de la vapeur dans le mélange) par nucléation homogène est un maximum de $S_{\mathrm{c}}$ et c'est cette valeur que nous avons utilisée et qui se trouve sur la figure $14 . S_{c}$ est alors une fonction de la température locale définie à partir du taux $I$ de formation des noyaux liquides par unité de temps et de volume [16]



Fig. 14. - Profils de sursaturation en fonction de l'abscisse réduite. Comparaison avec le taux de sursaturation critique. _ : Sursaturation locale pour une surchauffe donnée; - . - : Sursaturation critique à la température locale; $\Delta \Delta$ : Surchauffe $\Delta \theta=0{ }^{\circ} \mathrm{C} ;++$ : Surchauffe $\Delta \theta=5^{\circ} \mathrm{C}$.

[Supersaturation curves versus abscissa. Comparison with critical supersaturation ratio. - Local supersaturation; - - : Local critical supersaturation; $\Delta \Delta$ : $\Delta \theta=0^{\circ} \mathrm{C} ;++\Delta \theta=5^{\circ} \mathrm{C}$. $]$

$$
I=K \cdot \exp (-\Delta G / k \theta)
$$

où $K$ est un facteur lié aux fréquences de collision des molécules et $G$ est l'énergie de formation d'un noyau liquide, fonction du taux de sursaturation $s . S_{\mathrm{c}}$ est la valeur pour laquelle le taux $I$ devient égal à $1 \mathrm{~cm}^{-3} \mathrm{~s}^{-1}$

$$
I\left(S_{\mathrm{c}}\right)=1 \mathrm{~cm}^{-3} \mathrm{~s}^{-1} .
$$

Au-delà de $S_{\mathrm{c}}$, on montre que la nucléation homogène se produit à un rythme très élevé; $S_{\mathrm{c}}$ est donc considéré comme le seuil de déclenchement de la condensation.

Pour des cas du type 1 (Fig. 14), où la surchauffe $\Delta \theta$ de la vapeur est $5^{\circ} \mathrm{C}, s$ reste inférieur à $S_{\mathrm{c}}$, en tout point de l'écoulement et sous réserve des remarques ci-dessus, la condensation ne se produit qu'à la paroi, le système est stable et le calcul valable. Par contre, dans le cas 2, pour lequel la surchauffe $\Delta \theta$ est nulle, il existe des points où $s$ est supérieur à $S_{\mathrm{c}}$ et dans la mesure où $S_{\mathrm{c}}$ a été maximalisé, on peut affirmer qu'une condensation en volume aura lieu avec apparition de brouillard. Le calcul doit alors être repris en tenant compte de la saturation locale de la vapeur dans l'écoulement.

Ce résultat obtenu sưr un modèle simple permet d'expliquer les régimes distincts rencontrés au cours des expérimentations selon les conditions thermiques imposées : la figure 15 donne dans le plan $\theta_{\mathrm{s}}-\theta(l)$, $W_{\mathrm{m}}$ les courbes délimitant les régions avec ou sans brouillard pour une température de saturation de $100{ }^{\circ} \mathrm{C}$ et trois hypothèses de surchauffe $\Delta \theta\left(5^{\circ} \mathrm{C}\right.$, $0^{\circ} \mathrm{C}$ et $\left.-5^{\circ} \mathrm{C}\right)$. Le cas d'une surchauffe négative $\left(-5^{\circ} \mathrm{C}\right)$ correspond à un état sursaturé en $x=0$ (par exemple, après une détente). Comme pour les expériences, le brouillard apparaît soit lorsque la fraction massique moyenne $W_{\mathrm{m}}$ est élevée (supérieure à 0,3 ou 0,4 dans les cas étudiés où les écarts de température sont de l'ordre de $50^{\circ} \mathrm{C}$ ) soit lorsque la surchauffe de la vapeur injectée est faible. La maximalisation faite pour $S_{\mathrm{c}}$ indique que les courbes réelles sont probablement décalées vers l'origine par rapport à ces courbes théoriques. La zone, où les brouillards apparaissent, serait donc plus étendue.

\section{Conclusion.}

Bien que le calcul effectué ci-dessus le soit dans un cas idéal, il montre et l'expérience décrite au 2. le confirme, qu'a priori, tout problème de condensation avec incondensables peut mener selon les conditions thermiques de l'échange à une condensation limitée à la paroi ou, au contraire, affectant une zone de mélange au voisinage de celle-ci. Cela explique pourquoi certains auteurs $[5,9]$ ne considèrent que des condensations à la paroi alors que d'autres constatent expérimentalement l'existence de brouillards près des parois et supposent donc dans leurs calculs que la vapeur est saturée et humide dans tout l'écoulement [4]. 


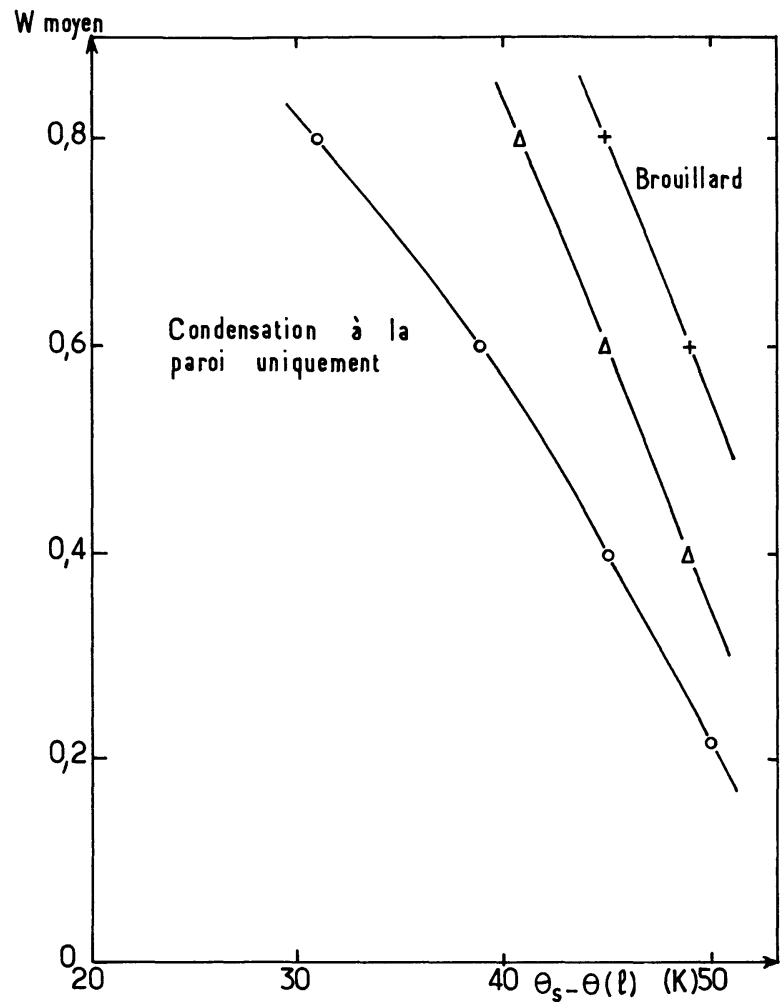

Fig. 15. - Domaines de condensation avec et sans brouillard pour trois valeurs de la surchauffe $\Delta \theta$. Température de la vapeur : $\theta(0)=100^{\circ} \mathrm{C}$. $\mathrm{O}-\mathrm{O}: \Delta \theta=-5^{\circ} \mathrm{C}$; $\triangle \Delta: \Delta \theta=0^{\circ} \mathrm{C} ;+-+: \Delta \theta=5^{\circ} \mathrm{C}$.

[Condensation zones with fogs for different values of $\Delta \theta$. ○- $\mathrm{O}: \Delta \theta=-5^{\circ} \mathrm{C} ; \Delta \triangle \Delta: \Delta \theta=0{ }^{\circ} \mathrm{C} ;+-+$ : $\Delta \theta=5^{\circ} \mathrm{C}$.]
Il apparaît que la démarche la plus raisonnable est de vérifier, après calcul, la présence ou l'absence de brouillards par l'intermédiaire des sursaturations locales. Cette démarche est cependant difficile dans les cas où la connaissance simultanée des fractions massiques et des températures nécessitent de lourds calculs. Lorsque les incondensables sont présents en faible quantité $-W_{\mathrm{m}}$ inférieure à $1 \%$ (Eq. (1)) - il n'y a pas lieu de considérer d'autre condensation que celle à la paroi ni d'autre flux que le flux condensé et la connaissance des températures locales n'est pas indispensable. Par contre, pour des fractions massiques moyennes élevées $-W_{\mathrm{m}}$ supérieur à $5 \%$ - il semble nécessaire de calculer le champ de températures afin de prévoir l'absence ou la présence de brouillards. De plus, lorsque des brouillards apparaissent, le calcul est singulièrement compliqué par le fait que seule une zone au voisinage de la paroi froide peut être occupée par un brouillard, le reste du mélange vapeur gaz restant sec et la frontière entre les deux régions étant à déterminer. Le calcul des transferts thermiques par condensation lorsque des incondensables sont présents en grande quantité reste donc un problème ouvert.

\section{Remerciements.}

Le manuscrit a été réalisé sur un ordinateur IBM 4341 muni d'une composeuse 4250.

Nous remercions la société IBM pour la mise à disposition de ce matériel.

\section{Bibliographie}

[1] Tanner, D. W., Pope, D., Potter, C. J. et West, D., Heat transfer in dropwise condensation at low stream pressures in the absence and presence of non condensable gas, Int. J. Heat Mass Transfer 11 (1968) 181-190.

[2] Kroger, D. G. et Rohsenow, W. M., Condensation heat transfer in the presence of a non condensable gas, Int. J. Heat Mass Transfer 11 (1968) 15-26.

[3] Henderson, C. L. and MarChello, J. M., Film condensation in the presence of a non condensable gas, J. Heat Transfer 91 (1969) 447-450.

[4] Bernard, J. J., Diep, G. B., Legay Desesquelles, F. et Prunet Foch, P., Proc. 6th International Heat Transfer Conference, Toronto, Canada, 7-11 août 1978, vol. 2, 335-339.

[5] Sparrow, E. M., Minkowycz, W. J. et Saddy, M., Forced convection condensation in the presence of noncondensables and interfacial resistance, Int. J. Heat Mass Transfer 10 (1967) 1829-1845.

[6] Citakoglu, E. et Rose, J. W., Dropwise condensation. Some factors influencing the validity of heat transfer measurement, Int. J. Heat Mass Transfer 11 (1968) 523-37.
[7] Sparrow, E. M. et Eckert, E. R. G., Effect of superheated vapor and non condensable gas on laminar film condensation, A.I. Ch.E. J. 7 (1961) 473-477.

[8] Sparrow, E. M. et LiN, S. H., Condensation heat transfer in the presence of a non condensable gas, J. Heat Transfer 86 (1964) 430-436.

[9] Minkowycz, W. J. et Sparrow, E. M., Condensation heat transfer in the presence of noncondensables, interfacial resistance, superheating, variable properties and diffusion, Int. J. Heat Mass Transfer 9 (1966) 1125-1144.

[10] Rose, J. W., Condensation of a vapour in the presence of a non condensing gas, Int. J. Heat Mass Transfer 12 (1969) 233-237.

[11] Poinsot, T., Thèse de Docteur-Ingénieur, Ecole Centrale des Arts et Manufactures (1983), Paris.

[12] BARSCHDORF, D., Carrier gas effects on homogeneous nucleation of water vapor in a shock tube, Physics of Fluids 18, no 5 (1975) 529-535.

[13] ReIST, R. H. et ReISs, H., Investigation of the homogeneous nucleation of water vapor using a diffusion cloud chamber, J. Chem. Phys. 59, no 2 (1973) 665-671. 
[14] KatZ, J. L., Condensation of a supersaturated vapor. The homogeneous nucleation of the $n$ alkanes, J. Chem. Phys. 52, no 9 (1970) 4733-4748.

[15] SmoliK, J. et VitoveC, J., Calculation of supersaturation profiles in a thermal diffusion cloud chamber in the unsteady state, Chem. Eng. J. 19 (1980) 105-111.
[16] Wegener, P. P. et Pouring, A. A., Experiments on condensation of water vapor by homogeneous nucleation in nozzles, Phys. Fluids 7, 3 (1964) 352-361. 361. 